\title{
Depressão e disfunção eretiva: uma investigação da comorbidade*
}

Gregory Branco Haertel**

\section{RESUMO}

Depressão e disfunção eretiva são patologias muito comuns. A primeira tem cerca de $15 \%$ de prevalência durante toda a vida. Já a prevalência de disfunção eretiva chega a 5\% em homens de 40 anos, aumentando para 15 a $25 \%$ quando a idade ultrapassa os 65 anos. Apenas os elevados índices de prevalência de ambas as patologias já indicariam uma elevada probabilidade de ocorrer comorbidade. Mais assustador este quadro se torna quando uma análise mais profunda nos mostra que a alta frequência da comorbidade entre disfunção eretiva e depressão não é apenas casual.

Este trabalho consistiu na coleta de informações através de revisão da literatura, no intuito de esclarecer quais os fatores envolvidos e de que formas ocorre a comorbidade entre depressão e disfunção eretiva.

Chegou-se à conclusão de que esta comorbidade pode ocorrer de algumas maneiras específicas, com diferentes fatores envolvidos e com tera-

* Monografia apresentada à Sociedade Brasileira de Sexualidade Humana (SBRASH) para conclusão do Curso de Pós-graduação em Terapia Sexual.

** Médico Psiquiatra. Terapeuta Sexual formado pela SBRASH. 
pêutica singular: uma das patologias sendo secundária à outra, diretamente relacionada àquela; o tratamento antidepressivo levando à disfunção eretiva; alguma outra doença cursando com sintomas depressivos e dificuldade eretiva; o uso de fármacos outros que não antidepressivos levando a ambas as patologias, e eventos vitais ou outros estressores sendo primariamente responsáveis por disfunção eretiva e sintomas depressivos. A diferenciação entre cada uma destas formas de instalação é crucial para o sucesso do tratamento.

\section{INTRODUÇÃO}

A vida do homem depende de mecanismos básicos para sua conservação, entre eles a iniciativa para agir, seja para defesa, seja para criação, e a capacidade de se reproduzir. A perda da iniciativa leva a uma diminuição da atividade prática, tornando o homem mais sujeito aos fracassos (risco para sua sobrevivência). A dificuldade para se reproduzir torna impossível a continuidade da vida através dos filhos (risco para a espécie). Sendo assim, patologias em qualquer destas áreas serão um risco para o bem estar do indivíduo, tornando-se mais graves quando da ocorrência de ambas em uma mesma pessoa, ou seja, quando há comorbidade entre depressão e alguma disfunção sexual.

Tanto a depressão quanto a disfunção eretiva são patologias muito freqüentes na sociedade atual. Estima-se que a prevalência da primeira seja de aproximadamente 5 a $15 \%$, quando observado apenas a idade adulta $a^{1,2}$, com incidência duas vezes maior em mulheres ${ }^{1}$. Já a prevalência de disfunção eretiva chega a $5 \%$ em homens de quarenta anos, aumentando para 15 a $25 \%$ quando a idade ultrapassa os 65 anos $^{3}$. Só pela alta prevalência de ambas já seriam freqüentes os casos de homens deprimidos e com disfunção erétil, sem qualquer relação etiológica entre as duas patologias. Mais assustador este quadro se torna quando uma análise mais profunda nos mostra que a alta freqüência da comorbidade entre disfunção eretiva e depressão não é apenas casual, mas que uma pode interferir na gênese da outra, bem como ambas podem ser decorrentes de uma terceira patologia ou secundárias a algum tratamento medicamentoso.

O entendimento desta complexa relação tornará o clínico mais seguro para a correta condução do tratamento, bem como o sucesso no mesmo será mais provável.

\section{OBJETIVO}

Investigar as várias formas de comorbidade entre depressão e disfunção eretiva no que se refere às formas de ocorrência e possíveis diretrizes terapêuticas. 


\section{DISFUNÇÃO ERETIVA EM HOMENS COM SINTOMAS DEPRESSIVOS}

Inicialmente é bom que seja esclarecido que a depressão não parece ser uma entidade única, por melhor que possa ter sido categorizada pelos instrumentos diagnósticos atualmente vigentes (DSM-IV e CID 10). Há depressões em que predominam sintomas subjetivos como desesperança, outros onde o que predomina é uma lentificação psicomotora, há aquelas em que ocorre diminuição do apetite e do sono, em outras o inverso é o que acontece. Sabese, também, que certos antidepressivos, com características distintas, podem ter um melhor efeito sobre determinado tipo de depressão (por exemplo, os IMAO sobre depressão atípica). Conclui-se, pois, que estamos diante de um quadro clínico composto por várias entidades distintas, provavelmente com substrato biológico que as diferencie e tendo, cada uma destas entidades, relações específicas com a esfera sexual. Desta forma, determinado "tipo" de depressão pode não ter qualquer influência direta sobre a sexualidade, enquanto outro "tipo" pode estar incontestavelmente ligado a estas disfunções.

Independente da discussão sobre os possíveis subtipos de depressão, que extrapola os nossos objetivos, o certo é que algumas pesquisas têm mostrado que a pessoa deprimida tem mais queixas de diminuição da satisfação sexual do que de diminuição do interesse ou da excitação ${ }^{6}$, o que ainda não encontra explicação no meio científico.

Além da já conhecida diminuição do desejo sexual, algumas pesquisas têm confirmado que o transtorno depressivo maior também está freqüentemente associado à diminuição da função eretiva e diminuição da atividade sexual ${ }^{7,8}$, sendo que, disfunção eretiva tem sido observada duas vezes mais em homens deprimidos a que em eutímicos?.

Várias pesquisas têm comparado valores de tumescência peniana noturna em homens com e sem transtorno depressivo maior e, apesar de certos resultados discordantes, trabalhos como o de Thase et al. ${ }^{7}$ indicam que a relação entre depressão e disfunção eretiva é importante. Neste trabalho demonstrou-se uma redução significativa no tempo de tumescência peniana noturna e na rigidez peniana em 34 homens deprimidos comparados com grupo controle.

Além do unicamente orgânico, é interessante frisar que um mínimo de desejo sexual auxilia na obtenção de uma ereção direcionada à atividade sexual, desejo este que está diminuído em indivíduos com sintomas depressivos.

Teoricamente, pessoas que desenvolvem disfunção eretiva devido a um quadro depressivo se beneficiam de tratamento antidepressivo exclusivo. Apesar desta melhora ocorrer na maioria dos casos, o uso de antidepressivos por si só pode induzir ou piorar sintomas sexuais, devendo o clínico estar atento a este fato.

Alguns autores sugerem uma relação neurofisiológica entre depressão e disfunção eretiva que seria responsável pela maior incidência desta última em homens com transtorno depressivo maior ${ }^{3}$, o que necessita de estudos mais aprofundados. 


\section{SINTOMAS DEPRESSIVOS EM HOMENS COM DISFUNÇÃO ERETIVA}

A relação entre disfunção sexual e algum diagnóstico psiquiátrico já tem sido estudada há muito, e, pelo menos dois estudos tem encontrado importante comorbidade psiquiátrica em pacientes de clínicas para tratamento de disfunções sexuais. Algo em torno de um terço destes pacientes apresentava algum diagnóstico psiquiátrico, principalmente transtornos afetivos (depressão ou transtorno bipolar), ansiosos, ou de personalidade ${ }^{10,11}$.

Alguns autores também demonstraram que depressão e sentimentos de raiva estavam bastante relacionados com disfunção eretiva ${ }^{3}$.

Vários estudos têm observado uma forte relação entre disfunção eretiva, baixa qualidade de vida e problemas no funcionamento social e ocupacional ${ }^{12}$, fatores também ligados a sintomas depressivos.

Em nossa sociedade, onde a noção de homem está intimamente ligada à noção de virilidade, de capacidade de ter um bom desempenho sexual, a falência deste "poder" (já chamada de impotência) leva a um constrangimento enorme, por vezes a um isolamento e não raramente a comportamentos autodestrutivos, até tentativas de suicídio. Estas atitudes e pensamentos mais relacionados à noção de potência perdida são, psicopatologicamente idênticas a certos sintomas depressivos, contribuindo por vezes para o aparecimento do quadro depressivo completo.

Um estudo recente de Seidman et al. ${ }^{13}$, duplo cego controlado com placebo, conduziu um grupo de 146 homens com diagnóstico de disfunção eretiva e sintomas depressivos não suficientes para o diagnóstico de transtorno depressivo maior pelo DSM-IV, metade dos quais usou entre 50 e $100 \mathrm{mg}$ de Sildenafil (Viagra) ao dia durante três meses. Houve melhora significativa de sintomas depressivos e de disfunção eretiva com o tratamento exclusivo com Sildenafil quando comparado com o grupo controle.

Este resultado, que necessita ser reproduzido, mostra-nos o quanto sintomas depressivos podem ser simplesmente secundários ao quadro disfuncional sexual, e que o tratamento da disfunção eretiva pode ter efeitos positivos sobre estes sintomas.

\section{TRATAMENTO DA DEPRESSÃO INDUZINDO DISFUNÇÃO ERETIVA}

Os antidepressivos são os medicamentos de eleição para o tratamento da depressão, sendo, porém, bastante heterogêneos quanto à forma de ação e efeitos colaterais. 
Todos os antidepressivos estão associados, em potencial, com efeitos colaterais sexuais. Aproximadamente 30 a $40 \%$ dos pacientes em uso de ISRS (a classe de antidepressivos mais utilizada no momento) terão algum tipo de disfunção sexual ${ }^{14}$. Estes efeitos adversos da medicação devem ser pesquisados objetivamente pelo médico, visto que a porcentagem de pacientes que os informa sem terem sido indagados é muito menor do que a real (de aproximadamente $7 \%$ para $55 \%$ quando perguntado objetivamente) $)^{15}$.

Como diferentes antidepressivos atuam em diferentes neurotransmissores, esse estudo deve ser feito separadamente para cada classe, já que distintos são também os seus efeitos sobre a sexualidade.

É preciso ter em mente também que os antidepressivos podem ter dois tipos de efeitos colaterais sexuais: primeiramente por sua ação no SNC, alterando os níveis dos neurotransmissores, mas também por sua ação no SNA (sistema nervoso autônomo), alterando níveis de noradrenalina e acetilcolina e incrementando ou diminuindo ações dos sistemas simpático e parassimpático .

A medicação antidepressiva não deve ser responsabilizada pela disfunção eretiva a menos que certos dados tenham sido esclarecidos. Deve ser pesquisado quando se deu o início da dificuldade sexual, pois como já foi visto, não são raros os casos em que esta se segue a um transtorno depressivo. Deve-se também pesquisar como está o relacionamento conjugal e se já existiram períodos de dificuldade eretiva no passado, mesmo na ausência de tratamento antidepressivo. Após este cuidadoso estudo pode-se tentar algumas manobras para observar se há melhora da disfunção eretiva como diminuição da dose da medicação ou feriado da droga e, em último caso, troca da classe da medicação antidepressiva. Havendo melhora é certo que a disfunção era devido à medicação psiquiátrica.

Disfunção eretiva tende a ser induzida mais por medicações que tenham forte efeito anticolinérgico como, por exemplo, os antidepressivos tricíclicos (ATC) e os inibidores da monoamino oxidase (IMAO), embora drogas que aumentam os níveis de serotonina como inibidores seletivos da recaptação da serotonina (ISRS) também possam causá-la.

Uma das possíveis classificações dos antidepressivos é a que dá importância ao tipo de neurotransmissor influenciado. É nesta que nos basearemos para que possamos entender como cada classe de droga age sobre a função sexual, notadamente sobre a fase de excitação.

\subsection{IMAO}

São drogas inibidoras da enzima monoamina oxidase, responsável pela metabolização das monoaminas centrais. Sua ação se dá pelo aumento da disponibilidade dessas monoaminas (principalmente noradrenalina e serotonina, mas também dopamina) em nível central ${ }^{16}$. Quando a inibição 
desta enzima é irreversível, e no uso de derivados do leite (tiramina) existe o risco de crises hipertensivas graves e com risco de vida (por aumento exagerado na disponibilidade de noradrenalina). Depois dos ISRS, os IMAO parecem ser os que mais efeitos colaterais sexuais possui ${ }^{14}$.

Os IMAO podem estar relacionados com inibição do desejo ${ }^{17}$, excitação ${ }^{18} \mathrm{e}$ orgasmo ${ }^{19}$, tanto em homens quanto em mulheres. A maioria dos estudos foi feito com uso de fenelzina em doses entre 60 e $90 \mathrm{mg} / \mathrm{dia}$. Tem sido sugerido que a moclobemida, um inibidor reversível da MAO A, tem efeito estimulante sobre a sexualidade em pessoas deprimidas, atuando sobre as três fases: desejo, excitação e orgasmo ${ }^{20}$.

\subsection{ANTIDEPRESSIVOS TRICÍCLICOS (ATC)}

São drogas clássicas no tratamento da depressão. Sua ação se dá pelo bloqueio das bombas de recaptação de serotonina e noradrenalina e, em menor grau, da bomba de recaptação de dopamina, ou seja, aumentando a disponibilidade destes neurotransmissores. Há também ação anticolinérgica, de antagonismo da histamina e de antagonismo alfa1 adrenérgico ${ }^{16}$.

Depois dos ISRS e dos IMAO são os mais relacionados com efeitos colaterais sexuais ${ }^{10}$. Podem estar relacionados com inibição do desejo ${ }^{21}$, excitação ${ }^{22} \mathrm{e}$ orgasmo ${ }^{21}$, tanto em homens quanto em mulheres. $\mathrm{ATC}^{21}$.

Ejaculação dolorosa foi encontrada em $20 \%$ dos homens em uso de

Tem sido observada, raramente, a associação de orgasmos espontâneos e bocejos no tratamento com clomipramina, o que deverá ser melhor estudado ${ }^{23}$.

A ação negativa destes medicamentos sobre a excitação, principalmente cursando com impotência sexual, deve ser por mecanismo anticolinérgico, uma vez que drogas anticolinérgicas são capazes de inibir os fenômenos vasocongestivos do tecido erétil do pênis ${ }^{5}$.

A ação inibitória sobre o desejo é provavelmente de origem serotoninérgica, o mesmo ocorrendo com relação ao orgasmo. Neste último, porém, principalmente no que se refere ao retardo ejaculatório, é possível haver concomitância de mecanismos serotoninérgicos e anticolinérgicos, visto que drogas anticolinérgicas também perturbam a fisiologia da ejaculação, pois, embora a primeira fase, ou fase de emissão, seja predominantemente simpática, a fase de ejaculação propriamente dita tem o seu neurocontrole exercido pelo sistema nervoso parassimpático e sistema nervoso voluntário 5 .

A clomipramina, um ATC de potente ação serotoninérgica, tem sido utilizada com sucesso no tratamento da ejaculação precoce ${ }^{24}$, talvez pela associação favorável dos efeitos serotoninérgicos e anticolinérgicos acima descritos. 


\subsection{ISRS}

Os inibidores seletivos da recaptação de serotonina são drogas mais recentemente utilizadas no tratamento da depressão. Tem vantagens no que se refere a sua maior especificidade e, conseqüentemente, menor número de efeitos colaterais. Apesar disto, no que se refere à esfera sexual, é a droga antidepressiva que possui a maior quantidade de efeitos adversos ${ }^{14}$.

Todos os antidepressivos desta classe causam disfunções sexuais, especialmente anorgasmia em mulheres e retardo ejaculatório em homens, em um número significativo de pessoas ${ }^{14}$.

O principal efeito sexual de medicamentos serotoninérgicos é sobre o orgasmo. Aproximadamente $10 \%$ das pessoas tratadas com fluoxetina apresentam anorgasmia ou retardo ejaculatório ${ }^{25}$, e uma porcentagem semelhante quando se usa paroxetina ou sertralina, sendo que, com estas duas últimas drogas este número diminui para cerca de $2 \%$ quando se trata de mulheres ${ }^{26}$.

Há também, em cerca de $10 \%$ dos pacientes tratados com fluoxetina, diminuição do desejo, sendo provável que com outras drogas serotoninérgicas ocorra o mesmo ${ }^{27}$.

A excitação é a fase menos comprometida, porém tem sido observado impotência na vigência do tratamento com estas drogas em um número significativamente maior do que o encontrado com uso de placebo ${ }^{27}$.

Em contraste com os dados acima expostos, sabe-se que antidepressivos com ação serotoninérgica (ISRS, IMAO, trazodona) podem, ocasionalmente, causar aumento da sexualidade ${ }^{28}$, o que é visto, infelizmente numa frequiência muito menor do que os efeitos anteriormente citados. Este aumento da libido parece estar, em alguns casos, associado com orgasmos espontâneos e bocejos ${ }^{28-31}$, um efeito ainda não plenamente entendido destas medicações (que já foi observado também com a clomipramina, um ATC com forte ação serotoninérgica).

Atualmente tem-se procurado usar os efeitos colaterais serotoninérgicos na esfera sexual de modo terapêutico, devido a sua grande freqüência. Assim, medicamentos com importante ação serotoninérgica como clomipramina, fluoxetina, paroxetina e sertralina têm sido uma opção segura no tratamento da ejaculação precoce ${ }^{32}$.

\subsection{BLOQUEADORES DA RECAPTAÇÃO DE NORADRENALINA E DOPAMINA}

São uma classe nova e aparentemente segura de antidepressivos, cuja principal droga, a bupropiona, tem sido utilizada com relativo sucesso em pessoas que desejam parar de fumar.

Não parecem estar associados à produção de incômodas disfunções sexuais como as que ocorrem com os ISRS, provavelmente por não apresentarem componente serotoninérgico significativo em seu mecanismo de ação ${ }^{16}$. 
Podem servir como boa opção na necessidade de trocar um antidepressivo por outro devido à presença de efeitos colaterais sexuais, já tendo sido visto diminuição das disfunções sexuais secundárias à medicação nesta mudança para a bupropiona ${ }^{33}$.

É bem possível que haja algum efeito sexual positivo devido a maior disponibilidade de dopamina central com esta medicação, o que merece novos estudos.

\subsection{INIBIDORES DA RECAPTAÇÃO DE SEROTONINA E NORADRENALINA}

Tem como principal representante a venlafaxina, que apresenta as mesmas propriedades de inibição da recaptação de noradrenalina e serotonina dos antidepressivos clássicos (tricíclicos), porém sem as propriedades de bloqueio dos receptores alfa1 adrenérgicos, colinérgicos ou histaminérgicos ${ }^{16}$.

Da mesma forma que com os ISRS, existe incidência aumentada de efeitos principalmente sobre o orgasmo (ejaculação retardada em $12 \%$ dos casos) e desejo, e em menor quantidade sobre a excitação (impotência em $6 \%$ dos casos) $)^{34}$.

\subsection{BLOQUEADORES SELETIVOS DA RECAPTAÇÃO DE NORADRENALINA}

Recentemente comercializada no Brasil, a reboxetina tem ação principalmente noradrenérgica, diferindo bastante das medicações serotoninérgicas vistas anteriormente. Não têm sido observados, até o momento, nenhum efeito significativo sobre o desejo ou o orgasmo. Impotência tem sido relatado numa incidência que varia entre 1,7 e $15 \%$. A maioria dos efeitos colaterais da reboxetina é de tipo anticolinérgico, o que talvez explique a elevada incidência de impotência.

\subsection{INIBIDORES DA RECAPTAÇÃO DE SEROTONINA + ANTA- GONISTAS DA SEROTONINA 2 (5HT-2)}

Este grupo é constituído por duas drogas novas, trazodona e nefazodona, que têm alterado bastante o conhecimento dos aspectos sexuais relacionados à depressão.

Ambas as drogas, além de inibidoras da recaptação de serotonina e antagonistas da 5HT-2, atuam como bloqueadoras alfa adrenérgicas, sendo que a trazodona possui efeitos mais potentes nestes receptores ${ }^{16}$. Talvez aí esteja a resposta para o aparecimento do priapismo como importante efeito colateral da trazodona, inexistente na nefazodona, que, aliás, até o momento, não tem apresentado qualquer evidência de efeitos colaterais sexuais ${ }^{35}$. 
Tem sido proposto que o uso da trazodona estaria associado a aumento da libido tanto em homens quanto em mulheres, bem como com aumento da excitação (ereção peniana e clitoriana ${ }^{36,37}$. Seu uso tem sido indicado principalmente em pessoas com depressão e impotência secundária.

Devido a sua ação serotoninérgica, ejaculação retardada também tem sido observada no uso da trazodona, bem como anorgasmia tanto em homens quanto em mulheres ${ }^{36}$.

\section{DEPRESSÃO E DISFUNÇÃO ERETIVA SENDO AMBAS CAUSADAS POR UMA TERCEIRA DOENÇA}

Algumas doenças podem causar, no seu curso, sintomas depressivos e sexuais, devendo o clínico estar atento quanto à gênese destes sintomas. Mais comumente sintomas depressivos e dificuldade eretiva são encontrados em endocrinopatas, sendo, porém, também observados em quadros neurológicos. Seis por cento das disfunções eretivas são causadas por endocrinopatias, normalmente devido à diminuição dos níveis de testosterona ${ }^{5}$. Diabetes é o fator endócrino mais importante na etiologia da disfunção eretiva, sendo que 40 a $60 \%$ dos pacientes diabéticos apresentam algum grau desta, não guardando relação com a gravidade da endocrinopatia ${ }^{5}$. No caso específico do diabetes a ação sobre o mecanismo eretivo não se dá via testosterona e sim por vasculopatias ou neuropatias no tecido erétil do pênis.

Outras endocrinopatias podem estar relacionadas com disfunção eretiva, destacando-se o hipotireoidismo, quando crônico (agudamente o hipotireoidismo tende a cursar unicamente com diminuição do desejo sexual); síndrome de Cushing e síndrome de Addison (a primeira pelo aumento dos níveis circulantes de cortisol, a segunda somente se houver diminuição da testosterona). Outras alterações hormonais também podem cursar com diminuição da função eretiva, notadamente a hiperprolactinemia, o aumento dos níveis de hormônio de crescimento $(\mathrm{GH})$ e os hipoginadismos.

Sintomas depressivos também podem estar relacionados com as endocrinopatias, tendo sido já descritos relação entre as síndromes de Cushing e Addison, os transtornos da paratireóide, da tireóide, o hiperaldosteronismo e a hiperprolactinemia com depressão ${ }^{4}$.

Conclui-se, pois, que o surgimento de uma endocrinopatia como, por exemplo, o hipotireoidismo, pode levar à gênese de disfunção eretiva e sintomas depressivos comcomitantemente, que tendem a remitir quando do tratamento adequado da endocrinopatia. Mais estudos são necessários quanto a esta comorbidade, porém o clínico de antemão deve estar atento à relação entre estas doenças. 
Outras causas médicas podem cursar mais raramente com sintomas depressivos e disfunção eretiva. Deve-se investigar patologias em sistema nervoso central (SNC), como tumores que, dependendo de sua localização, podem levar a ambos os sintomas.

Alcoolismo também deve ser pesquisado, visto a ver relação entre este e sintomas depressivos, bem como, através de neuropatias locais, dificuldade eretiva.

Muito raramente, para não se dizer nunca, apenas sintomas depressivos e disfunção eretiva são as manifestações da patologia subjacente. Deve-se, pois, pesquisar os demais sinais e sintomas característicos da síndrome ou da doença e, quando necessário, pedir exames complementares. O diagnóstico da patologia de base é fundamental visto que o tratamento da mesma tende a levar à remissão dos sintomas depressivos e sexuais.

\section{CAUSAS FARMACOLÓGICAS DE SINTOMAS DEPRESSIVOS E DISFUNÇÃO ERETIVA}

O uso de praticamente todas as drogas tem efeitos indesejáveis, ditos colaterais, associados ao efeito benéfico esperado pela medicação. Isto porque os fármacos tendem a ser absorvidos em maior ou menor grau por todo o organismo, e seus efeitos tendem a não ser tão específicos quanto o desejado.

Várias medicações podem ter o seu uso associado à sintomatologia depressiva e queixas de dificuldade eretiva, devendo o clínico basear-se em alguns parâmetros para saber se deve atribuir tais sintomas à medicação ou não. Em primeiro lugar deve-se ter bem claro quando a sintomatologia depressiva e erétil iniciou, para ver se há correlação cronológica entre o uso da droga e os ditos sintomas. Mesmo havendo relação cronológica, o clínico deve pesquisar se há fatores sócio-culturais, econômicos e familiares que possam explicar a disfunção erétil e a depressão. Para haver maior certeza diagnóstica, o clínico pode propor férias da medicação, por tempo limitado, observando sempre a meia vida do fármaco, para ver se os sintomas cessam ou não. Cessando os sintomas, e havendo recorrência dos mesmos quando do reinício da medicação, pode-se atribuir, com certeza, a sintomatologia ao uso da droga, devendo ser revista a necessidade de sua utilização.

Em princípio, praticamente todas as medicações podem causar efeitos colaterais nas esferas afetiva e sexual, se não pelos efeitos diretos (anticolinérgico, anti $\mathrm{B}$ adrenérgico), pela sensibilidade individual de quem a usa.

Medicações com importante efeito anticolinérgico, como certos neurolépticos, ou com forte efeito anti-B-adrenérgico (como a alfa metil dopa) tem elevada relação com disfunção eretiva ${ }^{5}$. 
Dentre as medicações que sabidamente estão associadas a sintomas tanto depressivos quanto de dificuldade eretiva destacam-se os neurolépticos (principalmente com efeito anticolinérgico como a Clorpromazina), certos hipotensores como a alfa metil dopa, os benzodiazepínicos quando em uso crônico, e a cimetidina $a^{4.5}$.

\section{DEPRESSÃO E DISFUNÇÃO ERETIVA CLINICAMENTE NÃO RELACIONADAS}

Inicialmente este subtítulo seria "depressão e disfunção eretiva etiologicamente não relacionadas", porém, sendo assim, este capítulo estaria restrito àqueles casos em que a disfunção eretiva ocorreria devido a uma condição (por exemplo, neuropatia) e a depressão a alguma outra, às vezes desconhecida. Nestes casos de etiologia não relacionada, obviamente o tratamento deverá ser feito de cada patologia individualmente, o que não impede um tratamento psicoterápico conjunto.

Interessa-nos mais os casos em que não há propriamente uma etiologia orgânica comum, mas sim fatores de vida que levam a sintomatologia depressiva e também disfunção eretiva.

É de importância fundamental a noção do termo "impotência", anteriormente utilizado para o que hoje se costuma chamar disfunção eretiva. Da mesma forma que o indivíduo com dificuldade eretiva sente-se "impotente", o deprimido também está "impotente" em relação à vida, ao prazer, ao significado. A depressão é uma dificuldade em experienciar o prazeroso como tal, ou seja, uma "impotência" em experienciar o prazer. Nada tem sido dito na literatura sobre a relação entre estas duas "impotências" do ponto de vista psíquico, apesar das várias hipóteses sugeridas para a gênese das disfunções eretivas (dificuldades familiares, econômicas, etc...). É provável que estes mesmos fatores também sejam importantes na comorbidade entre depressão e disfunção eretiva, o que carece de estudos.

\section{CONCLUSÃO}

A comorbidade entre depressão e disfunção eretiva é muito comum, sendo variadas as suas formas de ocorrência.

O clínico deverá estar atento à cronologia da instalação de cada doença para observar se há relação temporal entre ambas e se uma delas pode dever-se à presença inicial da outra. Neste caso sugere-se que seja tratada a doença base, sendo comum a remissão da sintomatologia total.

O uso de psicofármacos e outras medicações também deverá ser investigado, não sendo raro que disfunções eretivas sejam secundárias ao uso 
de antidepressivos, principalmente aqueles com importante ação anticolinérgica. A utilização de vários outros fármacos pode estar relacionada diretamente tanto à sintomatologia depressiva quanto a dificuldades eretivas, sendo possível que mesmo ambas as patologias sejam precipitadas pelo uso de alguma destas drogas.

Algumas doenças, como o hipotireoidismo, ou lesões em SNC podem estar relacionadas ao aparecimento de disfunções eretivas e sintomas depressivos, devendo o clínico observar outras alterações no exame físico e em exames complementares que sugiram estes quadros. É pouco provável que uma patologia qualquer tenha como únicos sintomas a dificuldade para manter a ereção e correlatos depressivos.

Eventos vitais negativos, dificuldades econômicas e sociais e questões psicológicas podem, sem exceção e por si só, causarem quadros comórbidos de depressão e disfunção eretiva.

Independente da forma de início da comorbidade, é muito importante que o clínico seja capaz de diferenciá-los entre si para uma melhor condução do caso. Salienta-se a necessidade de apoio psicológico e, muitas vezes, processos psicoterápicos, visto o impacto negativo que tais vivências, mesmo que temporárias, podem causar no desenvolvimento da pessoa.

\section{REFERÊNCIAS BIBLIOGRÁFICAS}

1. WEST, R. Depression. London, England: Office of Health Economics; 1992.

2. RICHELSON, E. Psychopharmacology 1993; 1 16(3): 461-478.

3. SEIDMAN, S. N.; ROOSE, S. P. The Relationship between Depression and Erectile Dysfunction. Current Psychiatry Reports 2000; vol 2, n² 3: 201-205.

4. KAPLAN, H. I.; SADOCK, B. J.; GREBB, J. A. Sinopsis of psychiatry. $7^{\text {th }}$ ed. Baltimore, Williams and Wilkins; 1994.

5. CAVALCANTI, R. Tratamento Clínico das Inadequações Sexuais. São Paulo, Brasil: Ed. Roca; 1992.

6. NOFZINGER, E. A.; THASE, M. E.; REYNOLDS, C. F. et al. Sexual function in depressed men: assesment by self-report, behaviorial and nocturnal penile tumescence measures before and after treatment with cognitive behavior therapy. Arch Gen Psychiatry 1993; 40: 24-30.

7. THASE, M. E.; REYNOLDS, C. F.; JENNINGS, R. et al. Nocturnal penile tumescence is diminished in depressed men. Biol Psychiatry 1988; 24: 33-46.

8. REYNOLDS, C. F. III; FRANK, E.; THASE, M. E. et al. Assesment of sexual function in depressed, impotent, and health men: factor analysis of a brief sexual function questionnaire for men. Psychiatry Res 1988; 24: 231-250.

9. ARAÚJO, A. B.; DURANTE, R.; FELDMAN, H. A. et al. The relationship between depressive simptoms and male erectile dysfunction: cross-sectional results from the Massachusets Male Aging Study. Psychosom Med 1998; 60: 458-465.

10. DEROGATIS, L. R.; MEYER, J. K.; KING, K. M. Psychopathology in individuals with sexual dysfunction. Am J Psychiatry 1981; 138: 757-763. 
11. FAGAN, P. J.; SCHMIDT, C. W.; WISE, T. N. et al. Sexual dysfunction and dual psychiatry diagnoses. Comp Psychiatry 1988; 29:278-284.

12. JONLER, M.; MOON, T.; BRANNAN, W. et al. The fact of age, ethnicity and geographical location on impotence and quality of life. $\mathrm{Br} \mathrm{J}$ Urol 1995; 75:651-655.

13. SEIDMAN, S.; ROSEN, R.; ROOSE, S. P. et al. Sildenafil citrate for erectile dysfunction and depression. XI World Congress of Psychiatry, Hamburg, Germany, August 1999.

14. GITLIN, M. J. Sexual side effects of psychotropic medications. In DUNNER, D. I.; ROSEMBAUM, J. F. (eds.). Psychiatric Clinics North Am Annual Drug Ther 1997; 4: 61-90.

15. MONTEJO, A. L.; LLORCA, G.; IZQUIERDO, J. A. et al. Sexual dysfunction wiht SSRis: A comparative analysis. New Research Programs and Abstracts of the Annual Meeting of the American Psychiatric Association, New York 1996; Abstract NR717: 226.

16. STAHL, S. M. Psychopharmacology of antidepressants. London, England: Martin Dunitz ed; 1997.

17. HARRISON, W. M.; RABKIN, J. G.; EHRHARDT, A. A. et al. Effect of antidepressant medication on sexual function: A controlled study. J Clin Psychopharmacol 1986; 144-149.

18. MITCHELL, J. E.; POPKIN, M. K. Antidepressant drug therapy and sexual dysfunction in men: A review. J Clin Psychiat 1983; 3(2): 76-79.

19. RAPP, M. S. Two cases of ejaculatory impairment related to phenelzine. Am J Psychiat 1979; 136: 1200-1201.

20. PHILIPP, M.; KOHNEN, R.; BENKERT. A comparison study of moclobemide and doxepin in major depression with special reference to effects on sexual dysfunction. Int J Clin psychopharmacol 1993; 7: 149-153.

21. BALON, R.; YERAGANI, V. R.; POHL, R. et al. Sexual dysfunction during antidepressant treatment. J Clin Psychiat 1993; 54: 209-212.

22. KOWALSKI, A.; STANLEY, R. O.; DENNERSTEIN, L. et al. The sexual side effects of antidepressant medication: A double-blind comparison of two antidepressants in a non psychiatric population. Br J Psychiat 1985; 147: 413-418.

23. MCLEAN, J. P.; FORSYTHE, R. G.; KAPKIN, I. A. Unusual side effects of clomipramine associated with yawning. Can J Psychiat 1983; 28: 569-570.

24. STRASSBERG, D. S.; DEGOUVEIA, C. A.; ROWLAND, D. L.; TAN, P.; SLOB, A. K. Clomipramine in the treatment of rapid (premature) ejaculation. J Sex Marital Ther, in press.

25. HERMAN, J. B.; BROTMAN, A. W.; POLLACK, M. H. et al. Fluoxetineinduced sexual dysfunction. J Clin Psychiat 1990; 51: 25-27.

26. Canadian Pharmaceutical Association. Compendium of pharmaceuticals and specialties. Ottawa, Canadá; 1995.

27. JACOBSEN, F. M. Fluoxetine-induced sexual dysfunction and an open trial of yohimbine. J Clin Psychiat 1992; 53: 119-122.

28. GARTRELL, N. Increased libido in women receiving trazodone. Am J Psychiat 1986; 143: 781-782.

29. MODELL, J. G. Repeated observations of yawning, clitoral engorgement, and orgasm associated with fluoxetine administration. J Clin Psychopharmacol 1989; 9: 63-65 (letter). 
30. SMITH, D. M.; LEVITTE, S. S. Association of fluoxetine and return of sexual potency in three elderly men. J Clin Psychiat 1993; 54: 317-319.

31. SULLIVAN, G. Increased libido in three men treated with trazodone. J Clin Psychiatry 1988; 49: 202-203.

32. BALON, R. Antidepressants in the treatment of premature ejaculation. J Sex Marital Ther 1996; 22(2): 85-96.

33. GARDNER, E. A.; JOHNSTON, J. A. Bupropion: An antidepressant without sexual pathophysiological action. J Clin Psychopharmacol 1985; 5: 24-29.

34. MONTVALE, N. J. Physicians desk reference. Medical Economics 1995.

35. FONTAINE, R.; ONTIVEROS, A.; ELIE, R. et al. A double-blind comparison of nefazodone, imipramine and placebo in major depression. J Clin Psychiat 1994; 55: 234-241.

36. BROGDEN, R. N.; HELL, R. C.; SPEIGHT, T. M.; AVERY, G. S. Trazodone: a review of its pharmacological properties and therapeutic uses in depression and anxiety. Drugs 1981; 21(6): 401-476.

37. VAN BEMMEL, A. L.; BEERSMA, D. G.; VAN DEN HOOFDAKKER, R. H. Changes in Eeg power density of non REM sleep in depressed patients during treatment with trazodone. J Affect Disord 1995; 35(1-2): 11-19. 